\title{
A Abordagem Estrutural das Representações Sociais e o Modelo dos Esquemas Cognitivos de Base: Perspectivas Teóricas e Utilização Empírica
}

\author{
Rafael Pecly Wolter ${ }^{1}$ \\ Departamento de Psicologia Social e Institucional da Universidade do Estado \\ do Rio de Janeiro, Rio de Janeiro, RJ, Brasil \\ Programa de Pós-Graduação em Psicologia da Universidade Salgado de Oliveira, \\ Niterói, RJ, Brasil \\ João Wachelke \\ Instituto de Psicologia da Universidade Federal de Uberlândia, Uberlândia, MG, Brasil \\ Denis Naiff \\ Instituto de Educação e Departamento de Psicologia da Universidade Federal Rural \\ do Rio de Janeiro, Seropédica, RJ, Brasil
}

\begin{abstract}
Resumo
A abordagem estrutural das representações sociais é amplamente utilizada em pesquisas brasileiras, no entanto, existe o uso quase exclusivo da análise prototípica (ou análise das evocações livres). O modelo dos esquemas cognitivos de base, elaborado por Rouquette e Guimelli estuda, dentro da abordagem estrutural, as relações que ocorrem no pensamento social. Mais precisamente, este modelo estuda as relações entre os diferentes cognemas ativados por um objeto de representação. As relações são formalizadas por 28 conectores reagrupados em três famílias ou meta-esquemas; avaliação, descrição e prática. O modelo prevê a utilização de três índices, ou valências, para descrever o grau de ativação de cada meta-esquema na população estudada o que nos permite diagnosticar a forma de pensamento acerca do objeto. Mais precisamente as valências indicam as dimensões representacionais ativadas pelo o objeto na população. Estas dimensões podem ser descritivas, ligadas ao julgamento e avaliação ou relacionadas com as práticas do grupo em questão.
\end{abstract}

Palavras-chave: Esquemas cognitivos de base, representações sociais, abordagem estrutural, núcleo central.

\section{The Basic Cognitive Schemes: Theorical Perspectives and Empirical Research}

\begin{abstract}
The structural approach to social representations is widely employed in Brazilian research. However, the studies use prototypical analysis (or free evocations analysis) almost exclusively. The basic cognitive schemes model proposed by Rouquette and Guimelli studies the relationships that take place in social thinking. More precisely, such model studies the relationships between the different cognems
\end{abstract}

Endereço para correspondência: Universidade do Estado do Rio de Janeiro, Rua São Francisco Xavier, 524/10 andar, sala 10.006, bloco B, Maracanã, Rio de Janeiro, RJ, Brasil 20550-900. E-mail: rafaelpeclywolter@ gmail.com,wachelke@yahoo.comednaiff@ufrrj.br

Financiamento: Fundação de Amparo à Pesquisa do Estado do Rio de Janeiro (FAPERJ). 
activated by a representation object. The relationships are formalized by 28 connectors regrouped in three families or meta-schemes; evaluation, description and practice. The model foresees the employment of three indexes, or valences, to describe the activation degree of each meta-scheme in the studied population, which makes it possible to diagnose the way of thinking about the object. These indexes aim at the dimension activated by the social object in the population. More specifically the indexes gives information about the presence of a descriptive dimension, of judgments and evaluation, and, at last, of social practices within the group.

Keywords: Basic cognitive schemes, social representations, structural approach, central core.

\section{Esquemas Cognitivos de Base: Perspectivas Teóricas y Investigaciones Empiricas}

\section{Resumen}

El abordaje estructural de las representaciones sociales es ampliamente utilizado en investigaciones brasileñas, al punto que existe el uso casi exclusivo del análisis prototípico (o análisis de las evocaciones libres). El modelo de los esquemas cognitivos de base, elaborado por Rouquette y Guimelli, estudia dentro del abordaje estructural, las relaciones que se presentan en el pensamiento social. Más precisamente, este modelo estudia las relaciones entre los diferentes cognemas activados por un objeto de representación. Las relaciones son formalizadas por medio de 28 conectores, reagrupados em tres familias o metaesquemas: evaluación, descripción y práctica. El modelo prevé la utilización de tres índices o valencias para describir el grado de activación de cada metaesquema en la población estudiada, lo que nos permite diagnosticar la forma de pensamiento en relación con el objeto. Mas precisamente, nos permite tener informaciones sobre las dimensiones representaconales activadas: descritiva; los juízos y avaliaciones; la práxis de las personas estudiadas.

Palabras clave: esquemas cognitivos de base, representaciones sociales, abordaje estructural, núcleo central.

A abordagem estrutural das representações sociais evoluiu extremamente, do ponto de vista teórico, no final dos anos 1980 e início dos anos 1990. A teoria do núcleo central, que é marcante na abordagem estrutural, a despeito de ter começado a surgir na tese de Abric (1976) foi desenvolvida e teve suas publicações marcantes nos anos 80 e 90 (Abric, 1987, 1994; Lheureux, Rateau, \& Guimelli, 2008; Rateau, 1995a, 1995b, 1995c; Sá, 1996). Outra teorização importante da abordagem estrutural, que relaciona práticas e representações, também teve seu auge de desenvolvimento teórico neste mesmo período, com o já clássico estudo de Christian Guimelli sobre a mudança de representação a partir de novas práticas dos caçadores (1989) e em 1994 o trabalho sobre os efeitos de novas práticas de enfermagem sobre a representação desta profissão. Neste mesmo ano,
1994, Claude Flament apresentou seu esquema sobre a mudança representacional a partir das práticas, descrevendo como a estrutura representacional busca, repetidamente e de diversas formas, se adequar a mudança de ação no grupo, para que esta mudança fique conforme ao pensamento. Podemos também citar os avanços teóricos da abordagem estrutural sobre como as representações se adequam às exceções e novidades (Guimelli, 2002; Guimelli \& Rouquette, 1993; Rouquette \& Guimelli, 1995; Wolter \& Rouquette, 2010). Poderíamos também citar o início, em 1988, dos avanços nos estudos sobre os objetos extremamente afetivos, ou nexus (Campos \& Rouquette, 2000; Rouquette, 1988, 1994; Wolter, 2009), e suas implicações nas relações grupais (Lo Monaco, Rateau, \& Guimelli, 2007) e avaliações de assuntos mobilizadores de massas (Wolter \& Rouquette, 2006, 
2010). Em relação às técnicas de estudo das representações sociais este período foi fértil em inovações com o surgimento da mise-en-cause (Moliner, 1989; Sá, 1996), da análise prototípica (Sá, 1996; Vergès, 1992), da indução por cenário ambíguo (Moliner, 1993) e do ressurgimento da análise de similitude (Flament, 1981, 1994). Inserido neste cenário, surgiu o modelo que descreveremos neste trabalho para propor desenvolvimentos técnicos e novas formas de utilização: os esquemas cognitivos de base.

\section{As Técnicas de Estudo da Abordagem Estrutural que Estudam a Relação entre o Objeto e o Cognema}

O campo das representações sociais é tradicionalmente plurimetodológico, comportando, por exemplo, desde estudos etnográficos (Jodelet, 1989) até experimentais (Abric, 1976; Rouquette, 1994). As formas de coleta de dados também são extremamente variadas com o uso de questionários (Gruev-Vintila \& Rouquette, 2007; Moscovici, 1976), entrevistas (Jodelet, 1989; Naiff \& Sá, 2007), associação livre de palavras (Sá, 1996; Vergès, 1992; Wolter \& Wachelke, 2013), grupos focais (Flick, 2004) ou documentos oriundos da mídia impressa (Moscovici, 1976). Por sua vez as análises de dados também traduzem a pluralidade do campo e variam da análise de discurso (Gomes \& Oliveira, 2012) à álgebra booleana (Flament \& Rouquette, 2003) passando pela análise de conteúdo (Bardin, 1977).

A abordagem estrutural das representações sociais possui técnicas que lhe são específicas e, com exceção da análise prototípica (Sá, 1996; Vergès, 1992; Wachelke \& Wolter, 2011), quase nunca usadas pela comunidade científica brasileira.

A teoria do núcleo central (Abric, 1994; Sá, 1996) descreve a representação social como um conjunto de cognemas, ou elementos cognitivos básicos (Codol, 1969), compartilhados por uma população, que dão sentido a um objeto do cotidiano. Estes cognemas, ativados pelo objeto, se relacionam entre si e formam uma totalidade. Dentre os cognemas, alguns são essenciais, ou absolutos, nos termos de Claude Flament (1994), para o reconhecimento e o pensamento acerca do objeto, pois sem eles o conjunto não é aceitável como conforme ao objeto para o grupo em questão. Estes cognemas, ou elementos, absolutos se associam a muitos outros cognemas por isso foram denominados de centrais para a representação social. Por serem absolutos e densamente ligados aos outros cognemas, estes elementos centrais são extremamente estáveis e pouco maleáveis e só se modificam com alterações de grande porte no contexto social do grupo e na sociedade. Por sua vez, existem outros elementos representacionais que são, segundo Flament (1994), negociáveis. Os membros do grupo toleram pensar no objeto sem que estes elementos estejam presentes pois eles são maleáveis, não necessariamente consensuais e menos associados a outros elementos comparativamente aos cognemas centrais.

Existem diversas técnicas que distinguem os elementos periféricos dos centrais, todas de alguma forma, estudam relações.

\section{Técnicas de Estudo da Relação Objeto e Cognema}

Dentre as técnicas para se distinguir o núcleo central (conjunto de cognemas centrais) da periferia muitas se interessam direta ou indiretamente, na relação entre os cognemas ativados e o objeto representacional.

A técnica mais difundida no Brasil, a análise prototípica, estuda este tipo de relação. Os diferentes participantes da pesquisa (em geral mais de cem), evocam o que lhes vêm à mente ao se deparar com o objeto representacional. Conforme afirmam Wachelke e Wolter (2011, p. 522)

as respostas fornecidas pelos participantes têm duas de suas coordenadas calculadas: a frequência no corpus do grupo e a ordem média de evocação, isto é, o valor resultante de uma média em que o valor 1 é atribuído para a resposta que é fornecida em primeiro lugar, 2 para a segunda resposta fornecida 
pelo participante, e assim por diante; como variante, há a possibilidade de pedir para os participantes realizarem uma classificação de importância, sendo atribuído valor 1 para a resposta mais importante, 2 para a segunda mais importante, etc. (Abric, 2003).

Por estudar o que é ativado quando o participante pensa no objeto a análise prototípica estuda a relação objeto-cognema a partir de dois critérios de acessibilidade do cognema (neste caso na forma de evocação): (a) sua frequência de ativação na população estudada; (b) a velocidade de sua ativação na população estudada.

A técnica do questionamento, ou mise-en-cause, também estuda a relação objeto-cognema mas sob outro aspecto; o da negociabilidade do cognema para o reconhecimento do objeto representacional. Esta técnica consiste em apresentar cenários onde os elementos representacionais candidatos ao núcleo central estão presentes com exceção de um dos candidatos. Os participantes devem dizer se reconhecem ou não o objeto representacional. Por exemplo, em diversos estudos (Moliner, 1989; Rateau, 1995c) o grupo ideal é visto como sendo composto por "pessoas amigas" (cognema 1), que "andam juntas" (cognema 2), "iguais" (cognema 3 ) e que possuem "as mesmas opiniões" (cognema 4). $\mathrm{Na}$ técnica do questionamento basta apresentar um grupo com três das quatro características e perguntar se os sujeitos consideram que o exemplar apresentado é o grupo ideal. Toda vez que sem a presença de um dos cognemas os sujeitos considerarem o objeto irreconhecível, é que este cognema é central. Neste exemplo, quando o cenário é apresentado com um grupo de pessoas que "andam juntas" (cognema 2), são "iguais" (cognema 3) e possuem "as mesmas opiniões" (cognema 4) mas não são "amigas" (cognema 1), os participantes em massa rejeitam este grupo como sendo ideal (Lheureux et al., 2008; Moliner, 1989; Rateau, 1995a). Em consequência, como este elemento amizade (cognema 1) é necessário e absoluto para o reconhecimento do objeto, logo ele é considerado central na representação do grupo ideal.

\section{Técnicas de Estudo da Relação entre Cognemas Representacionais}

Outra forma de se estudar as representações sociais dentro da abordagem estrutural consiste no enfoque à conexidade dos elementos. Por trás deste tipo de estudos existe a visão de que o elemento central da representação social é aquele que se relaciona intensamente com outros elementos representacionais. Como o núcleo central dá o sentido à representação, mais tecnicamente é possível afirmar que os elementos periféricos têm sua significação atrelada aos elementos centrais. Por exemplo, se no caso do estudo da representação social da aids para um grupo X o elemento periférico “jovens” está associado ao elemento central morte enquanto que para o grupo $\mathrm{Y}$ este mesmo elemento "jovens" está associado ao elemento sexualidade, teremos então duas interpretações completamente distintas do sentido de "jovens". Para o grupo X a AIDS seria uma doença que "mata jovens", por sua vez o grupo Y veria a AIDS como uma doença que "aparece pela sexualidade dos jovens". Nestes casos fictícios foi possível ver que a relação entre os elementos "jovem" e "sexualidade" ou "morte" é tão importante quanto a aparição dos elementos. Para estudar este tipo de relação o grupo do Midi difundiu a técnica da análise de similitude (Flament, 1981). Esta análise estuda as distâncias ${ }^{2}$ entre os diferentes elementos representacionais. Estas distâncias, tipicamente, são apresentadas em formas de árvores onde as ramificações traduzem as distâncias e os pólos são os elementos representacionais. Os elementos que são próximos de muitos outros elementos tendem a ser considerados centrais. Por sua vez os elementos próximos de poucos outros elementos tendem a ser considerados periféricos.

2 Existem diferentes maneiras de se calcular a distância entre elementos. A forma de calcular depende do tipo de coleta de dados e do algoritmo usado. 


\section{O Modelo dos Esquemas \\ Cognitivos de Base \\ e a Formalização das Relações entre Cognemas}

O modelo dos esquemas cognitivos de base (SCB) tem suas raízes na cibernética e se propõe a formalizar relações. No caso das representações sociais, Guimelli e Rouquette (1992) propuseram este modelo para estudar os tipos de relações possíveis entre elementos. Os estudos com a análise de similitude sempre foram úteis para o estudo da estrutura representacional. Entretanto, se esta análise indica a proximidade en- tre elementos, ela carece de informações sobre a forma como eles se relacionam. Por esta razão Christian Guimelli e Michel-Louis Rouquette (1992) resolveram adaptar o modelo dos SCB para o estudo dos tipos de relação entre elementos representacionais.

Primeiramente, é necessário dentro do modelo SCB conceber a representação social como um conjunto de elementos que se ligam por relações. Cada relação entre cognemas pode ser descrita com o triplet A R B, ou seja, o cognema A se relaciona com o cognema $\mathrm{B}$. Esta relação $\mathrm{R}$ pode tomar inúmeras formas como apresentadas na Tabela 1.

Tabela 1

Exemplos de Relação entre o Objeto e a Evocação

\begin{tabular}{cccc}
\hline Tipo de relação & Elemento A & Elemento B & Comentário \\
\hline Oposição & Guerra & Paz & Guerra é o antônimo de Paz \\
Definição & Guerra & Conflito armado & Guerra pode ser definida como um conflito \\
Classe que inclui & Carro & Gurgel & Gurgel é um tipo de carro \\
Classe incluída & Carro & Veículo & Carro é um tipo de veículo \\
Utilização & Motorista & Carro & Motorista utiliza o carro \\
\hline
\end{tabular}

Podemos compreender estas relações como caminhos cognitivos que levam de um elemento ao outro. Se, por exemplo, ao pensar no cognema Fluminense o grupo pensar em time de futebol, diversos caminhos podem ter sido percorridos:

- Fluminense é um tipo de time de futebol, o que traduz uma relação de classe incluída;

- Fluminense pode ser definido como um time de futebol, o que se traduz como uma relação definitória;

- Fluminense utiliza seu time de futebol (por exemplo, para arrecadar dinheiro ao clube), o que traduz uma relação de utilização;
- O Fluminense é sempre caracterizado por ser um time de futebol, o que traduz uma relação de caracterização.

Como estes quatro exemplos sobre a relação entre fluminense e time de futebol podem ilustrar, entre os dois cognemas existe mais de uma relação possível, em consequência existem diversos conectores que levam de um cognema ao outro. O modelo SCB formaliza, justamente, as diferentes relações possíveis entre dois elementos. Mais precisamente, segundo o modelo, 28 conectores (descritos na Tabela 2 ) relacionam dois conjuntos o que se traduz formalmente por A R28 B. 
Tabela 2

Meta-Esquemas e os 28 Conectores do Modelo dos Esquemas Cognitivos de Base

\begin{tabular}{|c|c|c|}
\hline $\begin{array}{l}\text { Dimensão } \\
\text { do } \\
\text { conector }\end{array}$ & $\begin{array}{c}\text { Nome dado ao } \\
\text { conector pelos } \\
\text { autores }\end{array}$ & Expressão padronizada na linguagem corrente \\
\hline \multirow{9}{*}{ Descrição } & $(\mathrm{SYN})$ & $A$ significa a mesma coisa, tem o mesmo sentido que $B$ \\
\hline & $(\mathrm{DEF})$ & $A$ pode ser definido como $B$ \\
\hline & $(\mathrm{ANT})$ & A é o contrário de $\mathrm{B}$ \\
\hline & (TEG) & $A$ faz parte de, é incluído em, é um exemplo de $B$ \\
\hline & (TES) & $A$ tem como exemplo, como caso particular, compreende, inclui $B$ \\
\hline & $(\mathrm{COL})$ & $A$ pertence à mesma classe (ou categoria) geral que $B$ \\
\hline & $(\mathrm{COM})$ & $A$ é um componente, um constituinte de $B$ \\
\hline & (DEC) & $A$ tem como componente, como constituinte $B$ \\
\hline & $(\mathrm{ART})$ & $A$ e $B$ são dois constituintes da mesma coisa (do mesmo objeto) \\
\hline \multirow{12}{*}{ Prática } & $(\mathrm{OPE})$ & $A$ faz $B$ \\
\hline & (TRA) & $A$ tem uma ação sobre $B$ \\
\hline & (UTI) & $A$ utiliza $B$ \\
\hline & $(\mathrm{ACT})$ & É $B$ que faz $A$ \\
\hline & $(\mathrm{OBJ})$ & $A$ é uma ação que tem como objeto, se exerce sobre, se aplica a $B$ \\
\hline & (UST) & Para fazer $A$, utiliza-se $B$ \\
\hline & $(\mathrm{FAC})$ & $B$ é alguém (uma pessoa, uma instituição) que age sobre $A$ \\
\hline & (MOD) & $B$ designa uma que se pode fazer sobre (a propósito de, no caso de, em relação a) $A$ \\
\hline & $(\mathrm{AOB})$ & $B$ é um instrumento que se utiliza sobre (a propósito de, no caso de, em relação a) $A$ \\
\hline & (TIL) & $A$ é utilizado por $B$ \\
\hline & (OUT) & Utiliza-se $A$ para fazer $B$ \\
\hline & $\mathrm{AOU}$ & $A$ é um instrumento que se pode utilizar para $B$ \\
\hline \multirow{7}{*}{ Avaliação } & (CAR) & $A$ é sempre caracterizado por $B$ \\
\hline & $(\mathrm{FRE})$ & $A$ é frequentemente caracterizado por $B$ \\
\hline & $(\mathrm{SPE})$ & $A$ é às vezes, eventualmente, caracterizado por $B$ \\
\hline & (NOR) & $A$ deve ter a qualidade de $B$ \\
\hline & $(\mathrm{EVA})$ & $A$ avalia $B$ \\
\hline & $(\mathrm{COS})$ & $A$ tem como causa, depende de $B$ \\
\hline & $(\mathrm{EFF})$ & $A$ tem como efeito (conseqüência ou fim), provoca $B$ \\
\hline
\end{tabular}

Estes 28 conectores foram agrupados em cinco famílias ou esquemas: Lexico (com 3 conectores), Vizinhança (3), Composição(3), Praxia (12) e Atribuição (7). Para Flament e Rou- quette $(2003$, p. 77$)$ os esquemas cognitivos de base correspondem a uma "estrutura de organização do conhecimento, que pode ser instanciada por uma diversidade indefinida de conteúdos". 
Segundo Guimelli (2003, pp. 121-122)

O SCB LEXICO agrupa os conectores lexicográficos:

- equivalência (syn): violência SYN brutalidade;

- oposição (ANT): violência ANT não violência;

- definição (DEF): violência DEF Força usada contra o direito.

O SCB VIZINHANÇA, agrupa os conectores que expressam as relações de inclusão ou de co-inclusão:

- a classe inclusa (TES): cidade TES rua;

- a classe incluidora (TEG): Rua TEG cidade;

- co-inclusão (COL): Cidade COL Aglomeração (explicar tradução)

O SCB COMPOSIÇÃO agrupa os conectores ao estabelecer as relações do todo à parte, da parte ao todo e da parte à parte:

- todo à parte (DEC): Natureza DEC Rio;

- parte ao todo (COM): Floresta COM natureza;

- Parte à parte (ART): Rio ART Floresta.

O SCB PRAXIA agrupa os conectores relacionados que se relacionam com a ação. Ele se organiza segundo a fórmula Ator*Objeto*Ferramenta (exemplo: "O caçador mata a presa com uma espingarda"). A partir desta fórmula, podemos compor 2 a 2 os elementos e teremos doze conectores: - "Fazer" (Ator OPE ação): Caçador OPE Matar;

- Simétrico: (ação ACT Ator): Matar Act Caçador;

- "Ter uma ação sobre": (Ator TRA Objeto);

- "Simétrico": (Objeto FAC Ator): Presa

FAC caçador;

- "Usar" (Ator UTI Ferramenta): Caçador

UTI Espingarda;

- "Simétrico" (Ferramenta TIL Ator):

Espingarda TIL Caçador;

- “Ação realizada sobre” (Ação OBJ Objeto): Matar OBJ Presa;

- "Simétrico" (Objeto MOD Ação): Presa MOD Matar;

- "Para ação é usada ferramenta" (Ação UST Ferramenta): Matar UST Espingarda;
- "Simétrico" (Ferramenta OUT Ação): Espingarda OUT Matar;

- "Ferramenta usada sobre" (Ferramenta AOU Objeto): Espingarda AOU Presa;

- "Simétrico" (Objeto AOB Ferramenta): Presa AOU Espingarda.

O SCB atribuição agrupa os conectores ligados ao julgamento e avaliação. Ao objeto A, eles fazem corresponder o atributo B. -

- $\mathrm{O}$ atributo $\mathrm{B}$ é uma característica permanente (CAR) de A: Grupo ideal CAR Relações amistosa;

- Característica frequente (FRE): Grupo ideal FRE Mesmas opiniões;

- Característica ocasional (SPE): Grupo ideal SPE Relações de ciúme;

- Característica normativa (NOR): Grupo ideal NOR Igualitário;

- Característica avaliativa (EVA): Grupo ideal EVA Agradável;

- $O$ atributo $B$ é ligado à causa, origem, um fator (COS): Grupo ideal COS igualitário;

- $\mathrm{O}$ atributo $\mathrm{B}$ é ligado à consequência, um efeito ou uma meta (EFF): Grupo ideal EFF Membros satisfeitos.

Os cinco esquemas cognitivos de base (Léxico, Vizinhança, Composição, Praxia e atribuição) foram reagrupados em três meta-esquemas (Rateau, 1995b), o que foi corroborado por uma série de estudos correlacionais (Rouquette \& Rateau, 1998). Estes estudos demostraram que as famílias Léxico, Vizinhança e Composição, nas respostas dos sujeitos, covariam grandemente. Esta correlação era previsível pois as três famílias correspondem aos conectores que descrevem o objeto e por isso este meta-esquema foi denominado DESCRIÇÃO. No fim o modelo SCB ficou com três meta-esquemas: Descritivo (que agrupou os esquemas Léxico, Vizinhança e Composição), Atribuição (que corresponde aos conectores do esquema atribuição) e Praxia (que corresponde aos conectores do esquema praxia).

\section{O Modelo SCB e as Dimensões do Pensamento Social}

Partimos do pressuposto, dentro do quadro teórico e conceitual do pensamento social 
(Rouquette, 1973), de que toda lembrança corresponde a um registro cognitivo que pode ser funcional, normativo ou descritivo. Tomando a representação social (Moscovici, 1976) e a memória social (Bartlett, 1932/1995; Halbwachs, 1994, 2006) como instâncias do pensamento social, propomos que a segunda, como a primeira, comporta três dimensões - prática, avaliativa e descritiva - que correspondem, respectivamente, aos três registros cognitivos postulados por Rouquette.

A dimensão prática (Abric, 1976; Guimelli, 2003) é relativa à "relação instrumental que os indivíduos entretêm com o objeto de representação. Na verdade esta dimensão pode ser considerada como em relação direta com as práticas sociais que os sujeitos desenvolvem em relação ao objeto" (Guimelli, 2003, p. 136). Esta dimensão corresponde ao registro funcional das cognições.

Por sua vez, segundo Guimelli (2003, p. 136), a dimensão avaliativa é "ligada aos valores, às normas ou a estereótipos fortemente salientes no grupo; ela permite que o grupo faça julgamentos relativos ao objeto. Esta dimensão é provavelmente marcada por fatores ideológicos e históricos". Ela corresponde ao registro normativo das cognições.

A dimensão descritiva corresponde à ativação de características, fatos e eventos, em suma, ao que constitui e define o objeto. Esta dimensão corresponde ao registro descritivo das cognições.
Logicamente os três meta-esquemas dos esquemas cognitivos de base correspondem às três dimensões aqui descritas. Ou seja, o Meta-esquema Atribuição corresponde ao registro avaliativo do pensamento, enquanto o meta-esquema Praxia corresponde ao registo funcional e, por último, o meta-esquema Descrição corresponde, logicamente, ao registro descritivo. Convém ressaltar que as dimensões não se excluem mutuamente, pois um mesmo objeto pode ver ativadas, por exemplo, três, duas, uma ou nenhuma das dimensões de lembrança.

Esta ideia pode ser resumida numa matriz (Tabela 3) onde distinguimos dois tipos de situações: a presença ou a ausência de uma dimensão. Ter uma dimensão presente significa que o objeto em questão ativou na população do estudo um registro cognitivo nesta dimensão. Por outro lado, a ausência da dimensão significa que o objeto não ativou o registro cognitivo correspondente na população estudada. Evidentemente a realidade é mais complexa que uma simples divisão dicotômica de cada dimensão, e se aproximaria de um continuum sem ruptura. Mesmo com esta ressalva, nos parece pertinente imaginar os objetos nos casos mais extremos, pois isto reduz o número de possibilidades, criando oito categorias de objetos "prototípicos", que podem servir como referência aos diferentes objetos encontrados nas pesquisas sobre o pensamento social.

Tabela 3

Os Oito Tipos de Objetos Possíveis em Função da Ativação de Cada Dimensão

\begin{tabular}{cccc}
\hline Tipo de objeto & $\begin{array}{c}\text { Dimensão descritiva/Meta- } \\
\text { esquema Descrição }\end{array}$ & $\begin{array}{c}\text { Dimensão funcional/ } \\
\text { Meta-esquema Praxia }\end{array}$ & $\begin{array}{c}\text { Dimensão normativa/ } \\
\text { Meta-esquema atribuição }\end{array}$ \\
\hline I & 1 & 1 & 1 \\
II & 1 & 1 & 0 \\
III & 1 & 0 & 1 \\
IV & 0 & 1 & 1 \\
V & 1 & 0 & 0 \\
VI & 0 & 1 & 0 \\
VII & 0 & 0 & 1 \\
VIII & 0 & 0 & 0 \\
\hline
\end{tabular}




\section{O Modelo SCB como Base para Pesquisas Empíricas}

Atualmente o modelo dos esquemas cognitivos de base é usado empiricamente de duas formas: primeiramente para se estudar o status, central ou periférico, dos elementos ou para descrever as dimensões ativadas por um objeto de pensamento social.

\section{O Modelo SCB para Estudar o Status Estrutural dos Elementos}

A ideia por trás do uso do modelo SCB para descrever o status estrutural de um elemento é relativamente simples: os elementos centrais por terem alto poder associativo ativarão muitos conectores comparativamente aos elementos periféricos.

Para isto o procedimento de coleta de dados previsto pelo modelo segue, segundo Guimelli e Rouquette (1992) e apresentado por Sá (1996), três etapas:

a) associação contínua: apresenta-se aos sujeitos uma palavra indutora e se lhes pede para dar o mais rapidamente possível, por escrito, três palavras ou expressões que lhes venham à mente. Obtêm-se assim três respostas R1, R2 e R3.

b) justificação das respostas: para cada uma das respostas R1, R2 e R3, os sujeitos devem em seguida explicitar, por escrito em uma ou duas frases, as razões pelas quais deram suas respostas.

c) análise das relações palavra indutora / palavra induzida: os 28 operadores definidos no modelo são apresentados aos sujeitos sob a forma de expressões padronizadas (a expressão formalizada relativa ao operador é traduzida em linguagem corrente e se torna mais compreensível para o sujeito ingênuo).

O sujeito deve então decidir se sim, não ou talvez, a expressão padronizada é o reflexo da relação que intervém entre a palavra indutora e sua própria resposta. As 28 expressões são apresentadas sucessivamente para R1, R2 e R3. (Guimelli \& Rouquette, 1992, p. 198; tradução de Sá, 1996, p. 141)
No caso de se estudar o status estrutural de um elemento este último vira o indutor. Logo o termo indutor que ativar muitos conectores terá uma conectividade forte com os outros elementos e será considerado central.

Logicamente para se testar a centralidade de elementos é necessário conhece-los, por isto o modelo dos esquemas cognitivos de base só pode ser usado quando uma pesquisa prévia já ocorreu. No caso da pesquisa acerca do regime militar seis elementos eram candidatos ao núcleo central: censura, ditadura, militares/soldados, repressão, rigidez e tortura.

Por exemplo no caso do estudo acerca das representações de estudantes acerca do Regime Militar (Wolter, Sá, \& Wachelke, 2013) os autores apresentaram a seguinte consigne:

Os alunos universitários, ao serem questionados sobre o tema Regime Militar, citaram com frequência o termo Censura. O Sr.(a) poderia dizer as 3 palavras ou expressões que lhe vêm logo à lembrança quando se fala de Censura no contexto do Regime Militar. O mesmo procedimento é usado para todos os candidatos ao núcleo central e nos fornece as seguintes informações:

- quantidade de conectores ativados por elemento candidato ao núcleo;

- quantidade de conectores do meta-esquema Descrição ativados por elemento candidato ao núcleo;

- quantidade de conectores do meta-esquema Praxia ativados por elemento candidato ao núcleo;

- quantidade de conectores do meta-esquema Atribuição ativados por elemento candidato ao núcleo.

\section{As Valências dos Meta-Esquemas}

Com cada uma das informações é possível elaborar um índice. Primeiramente existe a valência total $(\mathrm{Vt})$ que corresponde, para cada elemento, à proporção de conectores ativados em relação ao total de conectores possíveis de serem ativados. Este índice vai de 0 (onde nenhum conector foi ativado pelo elemento) a 1 (onde todos os conectores foram ativados para todos os sujeitos). 


\section{$V t=\frac{\text { quantidade de conectores ativados }}{28\left(n^{\circ} \text { de conectores dos } S C B\right) X n^{\circ} \text { de respostas associativas }}$}

No caso do Regime militar os vinte estudantes forneciam três cognemas a partir elemento indutor "censura" e nos informavam quais dos 28 conectores eram usados nesta associação. No total haviam 1680 conectores possíveis de serem ativados que correspondem a $20 * 3 * 28$ (20 sujeitos que evocaram 3 termos que possuem 28 conectores possíveis de serem ativados). Neste caso preciso do elemento "Censura" da RS do Regime Militar, 742 dos 1680 conectores foram ativados, ou seja, a valência total foi de 0,44 (para o termo censura $\mathrm{Vt}=742 / 1680=0,44$ ). Quando comparada com resultados de outras pesquisas (Rouquette \& Rateau, 1998) podemos notar que este índice é relativamente elevado pois $44 \%$ dos conectores foram ativados. A títu- lo de comparação o elemento Soldado/Militares ativou 570 dos 1680 conectores ( $\mathrm{Vt}=0,34)$.

Ao comparar a proporção de ativação e não ativação dos elementos "Censura" e "Soldado/ Militar" o resultado é significativo, $\mathrm{X}^{2}=33,1432$ (glib=03) a $p<0,01$. Ou seja, é possível afirmar que o elemento "Censura" da representação do Regime Militar é mais ligada aos outros elementos desta representação comparativamente ao elemento "Soldados/Militares", o que se traduz por valências totais distintas (0,44 para "censura" e 0,34 para "soldados/militares").

No caso do cálculo da Valência descritiva (Vd) é usado o mesmo procedimento que para o cálculo da Vt, no entanto, somente os nove conectores do meta-esquema descrição são levados em conta.

$$
V d=\frac{\text { quantidade de conectores descritivos ativados }}{9\left(n^{\circ} \text { de conectores descritivos }\right) X n^{\circ} \text { de respostas associativas }}
$$

No caso do exemplo precedente, com o elemento "censura" da representação do regime militar tendo sua centralidade testada com estudantes $(n=20)$ há 540 conectores do meta-esquema Descrição possíveis de serem ativados e os resultados obtidos foram de 214 conectores des- critivos de fato usados. Logo, para o elemento "Censura" a Vd é de 0,40 (214/540).

O cálculo da Valência Praxia $(\mathrm{Vp})$ segue o mesmo procedimento onde os conectores práticos ativados formam o nominador e o total de conectores práticos formam o denominador.

$$
V p=\frac{\text { quantidade de conectores práticos ativados }}{12\left(n^{\circ} \text { de conectores práticos }\right) \times n^{\circ} \text { de respostas associativas }}
$$

O elemento "Censura" do nosso exemplo podia ativar, na população de 20 universitários questionados, 720 conectores do meta- -esquema praxia e ativou de fato $328, \log 0$ $\mathrm{Vp}=328 / 720=0,46$.

A Valência Atribuição (Va) por sua vez é calculada da seguinte forma:

$$
V a=\frac{\text { quantidade de conectores avaliativos ativados }}{7\left(n^{\circ} \text { de conectores avaliativos) } X n^{\circ}\right. \text { de respostas associativas }}
$$



de Base: Perspectivas Teóricas e Utilização Empírica.

No caso aqui citado do elemento "Censura" há 420 conectores possíveis de serem ativados e os 20 participantes ativaram 200. Logo Va é de $0,48(200 / 420)$.
A Tabela 4 resume a quantidade de conectores ativados e possíveis de serem ativados para o elemento "censura" com 20 estudantes universitários e 3 evocações por sujeito.

Tabela 4

Valências Descrição, Praxia e Atribuição do Elemento “Censura” para Universitários (n=20)

\begin{tabular}{ccccc}
\hline $\begin{array}{c}\text { Elemento } \\
\text { Censura }\end{array}$ & $\begin{array}{c}\text { Conectores } \\
\text { do meta-esquema } \\
\text { descrição }\end{array}$ & $\begin{array}{c}\text { Conectores } \\
\text { do meta-esquema } \\
\text { praxia }\end{array}$ & $\begin{array}{c}\text { Conectores } \\
\text { do meta-esquema } \\
\text { atribuição }\end{array}$ & $\begin{array}{c}\text { Total } \\
\text { de conectores } \\
\text { ativados }\end{array}$ \\
\hline Ativados & 214 & 328 & 200 & 742 \\
Possíveis & 540 & 720 & 420 & 0,48 \\
Valência & 0,40 & 0,46 & 0,44 \\
\hline
\end{tabular}

Por sua vez o elemento "soldado/militares", como podemos ver na Tabela 5, ativou, em números absolutos, menos conectores dos meta-esquemas descrição e praxia comparativamente ao elemento "censura". Isto traduziria uma maior conectividade do elemento "censura" comparativamente ao elemento "soldado/militares" e está maior conectividade ocorre em duas dimensões de pensamento: descritiva e prática.

Tabela 5

Valências Descrição, Praxia e Atribuição do Elemento "Soldados/Militares" para Universitários (n=20)

\begin{tabular}{ccccc}
\hline $\begin{array}{c}\text { Elemento } \\
\text { Soldados/ } \\
\text { militares }\end{array}$ & $\begin{array}{c}\text { Conectores } \\
\text { do meta-esquema } \\
\text { descrição }\end{array}$ & $\begin{array}{c}\text { Conectores } \\
\text { do meta-esquema praxia }\end{array}$ & $\begin{array}{c}\text { Conectores } \\
\text { do meta-esquema } \\
\text { atribuição }\end{array}$ & $\begin{array}{c}\text { Total } \\
\text { de conectores } \\
\text { ativados }\end{array}$ \\
\hline Ativados & 160 & 217 & 202 & 579 \\
Possíveis & 540 & 720 & 420 & 0,48 \\
Valência & 0,30 & 0,30 & & 0,34 \\
\hline
\end{tabular}

\section{Considerações Finais}

Desde a publicação do livro "O Núcleo Central das Representações Sociais" de Celso Sá em 1996, centenas de pesquisas no Brasil, no campo das representações sociais, utilizaram a abordagem estrutural. No entanto, mesmo com a apresentação no livro de Sá de diversas técnicas da abordagem estrutural, pouquíssimas pesquisas foram além da análise prototípica. Esta análise prima pela facilidade de aplicação dos dados e de análise com o auxílio do software Evoc e serve como um primeiro levantamento da estrutura representacional mas de forma alguma serve para distinguir com segurança os elementos centrais dos periféricos. Diversas técnicas foram criadas nos últimos 25 anos para estudar a estrutura representacional, dentre elas os esquemas cognitivos de base. Este modelo, com auxílio das diferentes valências, estuda a conectividade dos diferentes elementos representacionais e serve como segunda etapa de pesquisa. Esta segunda etapa, no entanto, necessita de uma quantidade reduzida de sujeitos quando comparada com a análise prototípica. As valências permitem também conhecer o grau de conectividade dos elementos e nos informam sobre o peso das diferentes dimensões representacionais. A nível teórico, conforme descrevemos no início deste trabalho, é importantíssimo distinguir a di- 
mensão de pensamento que o grupo tem acerca do objeto, pois pensar de forma prática traduz uma relação diferente com o objeto se compararmos ao pensamento avaliativo. No primeiro caso existiriam práticas sociais extremamente envolvidas com o pensamento, no outro caso, do pensamento com forte dimensão avaliativa, são as normas e valores que guiam o conhecimento sobre o objeto. Este aspecto dos esquemas cognitivos de base permite um maior refinamento teórico das pesquisas ao fornecer informações sobre a dimensão representacional presente na relação entre o grupo e o objeto. De forma mais ampla, dentro do quadro do pensamento social (Wachelke, 2013), os SCB auxiliam no estudo de diversas instâncias de pensamento social e não se restringem a trabalhos que se restringem à teoria do núcleo central.

Esta técnica possui também algumas desvantagens. A primeira dentre elas consiste no fato de que sua aplicação requere dos sujeitos um alto grau de abstração. Nem sempre o respondente se disponibiliza a responder a questões que demandam tamanha reflexão. Muitas questões do modelo SCB não fazem sentido ao participante pois o conector não foi ativado. Por exemplo, o conector FRE que traduz a frequência não une os elementos Paz e Guerra, logo ao fazer a pergunta "Paz é com frequência Guerra" aos participantes, geramos uma questão que não faz sentido para muitos sujeitos o que, por sua vez, tem um custo cognitivo pois os sujeitos refletem sobre questões que não lhes fazem sentido. A segunda crítica que poderíamos fazer consiste no tempo de aplicação, dificilmente os sujeitos respondem em menos de trinta minutos. Este segundo inconveniente contribui para aumentar o cansaço cognitivo que resulta da primeira desvantagem. Por último, por ser uma técnica que confirma a centralidade dos elementos, ela não permite um levantamento do conteúdo representacional e é usada quando o pesquisador já conhece os $\operatorname{cog}$ nemas que poderiam vir a ser centrais, logo só é possível usá-la numa segunda etapa de pesquisa ou quando o objeto de representação já foi muito estudado para o grupo em questão.

Usualmente os esquemas cognitivos de base são utilizados, como proposto por Guimelli e
Rouquette (1992), no estudo das relações entre cognemas de uma representação social. Parece-nos que é possível utilizar este modelo diretamente na relação entre o objeto e o cognema representacional, mas para isto novas pesquisas serão necessárias para examinar a validade de tal procedimento.

\section{Referências}

Abric, J.-C. (1976). Jeux, conflits et représentations sociales (Thèse d'État, Université de Provence, Aix-en-Provence, France).

Abric, J.-C. (1987). Coopération, compétition et représentations sociales. Cousset, Suisse: DelVal.

Abric, J.-C. (1994). Représentations sociales: aspects théoriques. In J.-C. Abric (Ed.), Pratiques sociales et représentations (pp. 11-36). Paris: Presses Universitaires de France.

Bardin, L. (1977). L'analyse de contenu. Paris: Presses Universitaires de France.

Bartlett, F. C. (1995). Remembering: A study in experimental and social psychology. Cambridge, MA: Cambridge University Press. (Original work published 1932)

Campos, P. H. F., \& Rouquette, M.-L. (2000). La dimension affective des représentations sociales: deux recherches exploratoires. Bulletin de Psychologie, 53(4), 435-441.

Codol, J. P. (1969). Note terminologique sur l'emploi de quelques expressions concernant les activités et processus cognitifs en psychologie sociale. Bulletin de Psychologie, 23(280), 63-71.

Flament, C. (1981). L'analyse de similitude: une technique pour les recherches sur les représentations sociales. Cahiers de Psychologie Cognitive, 4, 357-396.

Flament, C. (1994). Structure, dynamique et transformation des représentations sociales. In J. C. Abric (Ed.), Pratiques sociales et représentations (pp. 37-57). Paris: Presses Universitaires de France.

Flament, C., \& Rouquette, M.-L. (2003). Anatomie des idées ordinaires. Comment étudier les représentations sociales. Paris: Armand Colin.

Flick, U. (2004). Uma introdução à pesquisa qualitativa. Porto Alegre, RS: Bookman. 
Gomes, A. M. T., \& Oliveira, D. C. (2012). O uso da metáfora nos estudos de representações sociais: Uma abordagem da análise do discurso francesa. Trabalho apresentado na $11^{\text {a }}$ Conferência Internacional de Representações Sociais, Évora, Portugal.

Guimelli, C. (1989). Pratiques nouvelles et transformation sans rupture des représentations sociales: la représentation de 1 achasse et de la nature. In J.-L. Beauvois, R.-V. Joule, \& J.M. Monteil (Eds.), Perspectives cognitives et conduites sociales: Vol. 2. Représentations et processos sociocognitifs (pp. 117-141). Cousset, Suisse: Del Val.

Guimelli, C. (1994). La fonction d'infirmière: pratiques et représentations sociales. In J.-C. Abric (Ed.), Pratiques sociales et représentations (pp. 83-107). Paris: Presses Universitaires de France.

Guimelli, C. (2002). Etude expérimentale du rôle de l'implication de soi dans les modalités de raisonnement intervenant dans le cadre des représentations sociales. Revue Internationale de Psychologie Sociale, 1, 129-161.

Guimelli, C. (2003). Le modèle des schèmes cognitifs de base: méthodes et applications. In J.-C. Abric (Ed.), Méthodes d'étude des représentations sociales (pp. 119-143). Ramonville Saint-Agne, France: Érès.

Guimelli, C., \& Rouquette, M. L. (1992). Contribution du modèle associatif des schèmes cognitifs de base à l'analyse structurale des représentations sociales. Bulletin de Psychologie, 45(405), 196-202.

Guimelli, C., \& Rouquette, M.-L. (1993). Note sur la formalisation des schèmes étranges dans l'étude des représentations sociales. Les Cahiers Internationaux de Psychologie Sociale, 19, 43-48.

Gruev-Vintila, A., \& Rouquette, M.-L. (2007). Social thinking about collective risk: How do risk-related practice and personal involvement impact its social representations? Journal of Risk Research 10(3-4), 555-581. doi:10.1080/13669870701338064

Halbwachs, M. (1994). Les cadres sociaux de la mémoire. Paris: Albin Michel.

Halbwachs, M. (2006). A memória coletiva. São Paulo, SP: Centauro.

Jodelet, D. (1989). Représentations sociales: un domaine en expansion. In D. Jodelet (Ed.), Les représentations sociales (pp. 31-61). Paris: Presses Universitaires de France.

Lheureux, F., Rateau, P., \& Guimelli, C. (2008). Hiérarchie structurale, condionnalité et normativité des représentations sociales. Les Cahiers Internationaux de Psychologie Sociale, 77, 41-55.

Lo Monaco, G., Rateau, P., \& Guimelli, C. (2007). Nexus, représentations sociales et masquage des divergences intra et intergroupes. Bulletin de Psychologie, 60(6), 581-592.

Moliner, P. (1989). Validation expérimentale de l'hypothèse du noyau central des représentations sociales. Bulletin de Psychologie, XLI, 759-762.

Moliner, P. (1993). ISA: l'induction par scénario ambigü. Une méthode pour l'étude des représentations sociales. Revue Internationale de Psychologie Sociale, 6(2), 7-21.

Moscovici, S. (1976). La psychanalyse son image et son public. Paris: Presses Universitaires de France.

Naiff, L. M., \& Sá, C. (2007). De mãe para filha, o legado da exclusão social: Um estudo de memórias autobiográficas. Memorandum, 13, 88-99.

Rateau, P. (1995a). Le noyau central des représentations sociales comme système hiérarchisé. Une étude sur la représentation du groupe. Les Cahiers Internationaux de Psychologie Sociale, 26(2), 29-52.

Rateau, P. (1995b). Dimensions descriptive, fonctionnelle et évaluative des représentations sociales. Une étude exploratoire. Papers on Social Representations, 4(2), 133-146.

Rateau, P. (1995c). Hiérarchie du système central des représentations sociales et processus de rationalisation de la mise en cause de ses éléments. Bulletin de Psychologie, XLIX(422), 73-87.

Rouquette, M.-L. (1973). La pensée sociale. In S. Moscovici (Ed.), Introduction à la psychologie sociale (pp. 299-327). Paris: Larousse.

Rouquette, M.-L. (1988). La psychologie politique. Paris: Presses Universitaires de France.

Rouquette, M.-L. (1994). Sur la connaissance des masses. Grenoble, France: Presses Universitaires de Grenoble.

Rouquette, M.-L., \& Guimelli, C. (1995). Les "canevas de raisonnement" consécutifs à la mise en cause d'une représentation sociale: essai de formalisation et étude expérimentale. Les 
Cahiers Internationaux de Psychologie Sociale, 28, 32-43.

Rouquette, M.-L., \& Rateau, P. (1998). Introduction à l'étude des représentations sociales. Grenoble, France: Presses Universitaires de Grenoble.

Sá, C. P. (1996). Núcleo central das representações sociais. Petrópolis, RJ: Vozes.

Vergès, P. (1992). L'évocation de l'argent: une méthode pour la définition du noyau central d'une représentation. Bulletin de Psychologie, $X L V(405), 203-209$.

Wachelke, J. (2013). Beyond social representations: The conceptual bases of the structural approach on social thinking. Interamerican Journal of Psychology, 47, 131-138.

Wachelke, J. F. R., \& Wolter, R. P. (2011). Critérios de construção e relato da análise prototípica para representações sociais. Psicologia: Teoria e Pesquisa, 27, 521-526. doi:10.1590/S010237722011000400017

Wolter, R. P. (2009). Le cas des objets à forte valence affective: la notion de nexus In M.-L. Rouquette (Ed.), La pensée sociale. Questions théoriques et recherches appliquées (pp. 59-72). Ramonville Saint-Agne, France: Érès.

Wolter, R. P., \& Rouquette, M.-L. (2006). A influência de certos termos socialmente salientes (nexus) e da imagem sobre a percepção de um objeto social. Revista Educação Pública, 15(29), 79-89.
Wolter, R. P., \& Rouquette, M.-L. (2010). Situations de crise et modes de raisonnement: effets de l'intensité du positionnement sur le choix d'un canevas de raisonnement. Les Cahiers Internationaux de Psychologie Sociale, 87, 479-481. doi:10.3917/cips.087.0479

Wolter, R. P., Sá, C. P., \& Wachelke, J. F. R. (2013). Relações entre o objeto de Representação, os elementos representacionais e definição de centralidade: Algumas questões teóricas. Trabalho apresentado na VIII Jornada Internacional e VI Conferência Brasileira sobre Representações Sociais, Recife, PE, Brasil.

Wolter, R. P., \& Wachelke, J. F. R. (2013). Índices de distribuição de evocações: Raridade, diversidade e comunidade de corpora de representações sociais. Revista de Psicologia: Teoria e Prática, 15, 119-129.

Recebido: 06/05/2015

Aceite final: 23/09/2015 